\title{
Placing and Recalling Virtual Items on the Skin
}

\author{
Joanna Bergstrom-Lehtovirta, Sebastian Boring, Kasper Hornbæk \\ University of Copenhagen, Denmark \\ \{joanna, sebastian.boring, kash $\} @$ di.ku.dk
}

\begin{abstract}
The human skin provides an ample, always-on surface for input to smart watches, mobile phones, and remote displays. Using touch on bare skin to issue commands, however, requires users to recall the location of items without direct visual feedback. We present an in-depth study in which participants placed 30 items on the hand and forearm and attempted to recall their locations. We found that participants used a variety of landmarks, personal associations, and semantic groupings in placing the items on the skin. Although participants most frequently used anatomical landmarks (e.g., fingers, joints, and nails), recall rates were higher for items placed on personal landmarks, including scars and tattoos. We further found that personal associations between items improved recall, and that participants often grouped important items in similar areas, such as family members on the nails. We conclude by discussing the implications of our findings for design of skin-based interfaces.
\end{abstract}

\section{Author Keywords}

Skin input; recall performance; interface design

\section{ACM Classification Keywords}

H.5.m. Information Interfaces and Presentation (e.g. HCI): Miscellaneous

\section{INTRODUCTION}

Touching the skin can be used as input for smart glasses [17], televisions or other remote displays [2, 12], mobile phones [11], and auditory interfaces [6, 14]. These interfaces do not display touch targets directly on the skin - users need to learn and recall where targets are located. We refer to such targets as virtual items.

Landmarks on the skin (such as joints, bumps, wrinkles, tattoos, and veins) can improve input performance with virtual items [2, 6, 8, 11, 14, 17]. For instance, users can tap more accurately on the fingertips of the index finger and thumb [5] and on the elbow and wrist [11] than on areas between those. However, previous work has examined only a small number of landmarks on local parts of the body (e.g., the palm in [2,

Permission to make digital or hard copies of all or part of this work for personal or classroom use is granted without fee provided that copies are not made or distributed for profit or commercial advantage and that copies bear this notice and the full citation on the first page. Copyrights for components of this work owned by others than ACM must be honored. Abstracting with credit is permitted. To copy otherwise, or republish, to post on servers or to redistribute to lists, requires prior specific permission and/or a fee. Request permissions from permissions@acm.org

CHI 2017, May 6-11, 2017, Denver, CO, USA.

Copyright is held by the owner/author(s). Publication rights licensed to ACM ACM ISBN 978-1-4503-4655-9/17/05 ...\$15.00.

http://dx.doi.org/10.1145/3025453.3026030
13], the forearm in $[3,11]$, and the fingertips in [5]). Therefore, it is unclear which kinds of landmarks across the hand and forearm can be used for placing virtual items, and what the recall performance of item locations on the skin is.

The skin and landmarks on it are highly personal. Scars, birthmarks, and tattoos can be associated with individuals' personal lives and memories (Figure 1). Research in HCI and memory has shown that users create personal semantics when placing items on physical locations, suggesting that these associations have positive effects on recall [15]. Studies on user-designed layouts on the skin, however, have used familiar interfaces and abstract items, such as numbers [11], keyboards [17], or directional keys [2]. These may limit creation of personal associations and encourage consistency with familiar layouts. Thus, personal strategies for placing items on the skin - including the use of landmarks and establishing associations between items and locations - have remained unexplored, as have their effects on recall.

We collected in-depth data in a study where participants placed 30 virtual items on their hand and forearm, and attempted to learn and recall the locations of those items. We interviewed the participants to analyse strategies used in placing the items; collected touch data of mapped items across the skin area to identify frequently used locations; and captured images of hands and arms to examine the types of landmarks at different item locations. We also investigated recall performance across the locations and the items. This paper contributes empirical data of mapping and recall performance on the skin, and discusses implications for designing skin-based interfaces.

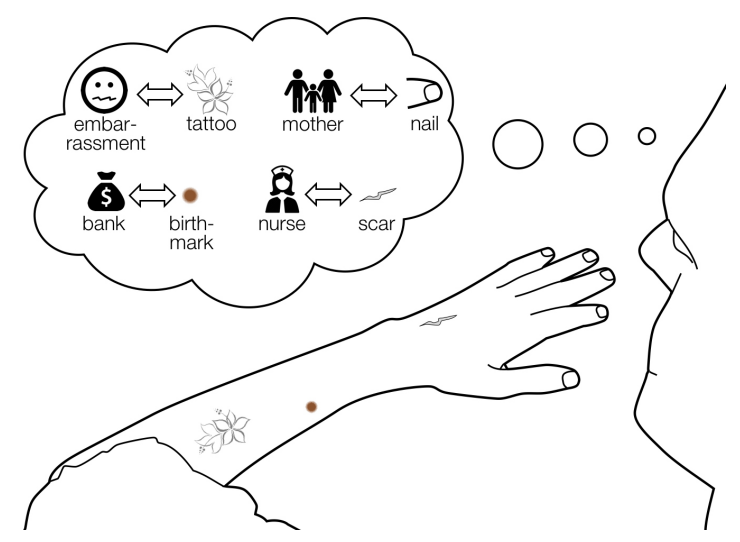

Figure 1: Some associations between the virtual items and locations on the skin that participants created in our study. 


\section{RELATED WORK}

The related work on skin-based interfaces consists of several studies using virtual items. Users can, for instance, browse through an audio-based mobile phone's menu by sliding a finger across invisible items on the fingers and palm [5], or type blind-folded on a personalized QWERTY-layout to give input for smart glasses [17]. Previous research has developed technologies to track touch input, measured touch accuracy on item locations, and examined how users design item layouts, such as what kind of landmarks they use on the skin.

\section{Technologies for Tracking Touch on the Skin}

Touch on bare skin can be estimated through optical tracking $[1,2,7,9,10,16,17]$ or by tracking signal propagation on the skin surface [8, 12]. Motion capture systems [7] and tracking of acoustic signals [8, 12] allow sensing touches across the entire forearm and hand areas. Infrared sensors are used locally for sensing touches; for instance, on the palm [9, 10, 17]. These technologies enable input on virtual items on the skin, with few restrictions on item layouts or the size of the interactive area. Therefore, it is important to examine where to place virtual items on both the forearm and hand.

\section{Touch Performance on Virtual Items}

Related work on skin input suggests that touch performance on virtual items is better when those items are located at landmarks on the skin, rather than on areas with fewer landmarks. For example, Gustafson et al. [5] used an imaginary grid layout with anchoring points on the thumb tip, the index fingertip, and the point where the thumb and index join, and showed that participants pointed most accurately on anchor points, while pointing error significantly increased the farther the target was from these landmarks. In another paper about imaginary interfaces, Gustafson et al. [6] demonstrated that in the absence of visual feedback from a palm interface, allowing users to see their bare hands still resulted in twice as fast target acquisition speed as in a blind-folded condition. Lin et al. [11] showed significantly higher tapping accuracies on locations at the elbow and wrist compared to three other locations along the forearm. These studies used a small number of targets, because their focus was on examining touch accuracy on the skin without visual feedback. To our knowledge, earlier studies have not measured recall performance of virtual item locations on the skin.

\section{Mapping Items on the Skin}

Related research has used landmarks to create interface layouts that fit the shape of the skin surface. Gustafson et al. [6], for instance, mapped mobile phone menu items on the hand and fingers using a layout similar to that on the phone. Instead of grid layouts that might suit such imaginary [5] or projected interfaces [7], researchers have also asked users to decide on or design virtual item layouts. For example, Lin et al. [11] asked participants to place a small number of targets (six to nine) on their forearm; Dezfuli et al. [2] asked participants to map TV remote control buttons on the palm side of the hand and fingers; and Wang et al. [17] asked participants to design a QWERTY -keyboard layout and adjust its key shapes for the palm side of the hand and fingers.
The virtual item placements participants made in these studies, however, may have been influenced by using existing, pre-defined layouts. For example, the directional keys of a TV remote control [2], and most keys of the QWERTY -keyboard [17] remained in similar locations in relation to each other on the palm and in the reference interfaces. Moreover, mapping abstract items - such as the numbers from 1-6 [11], or alphabetical keys [17] - can limit the creation of personal associations between the items and the skin, encouraging to maintain a familiar ordering between the items.

\section{Landmarks Around the Hand and Forearm}

Related work mentions various kinds of landmarks on the skin, such as freckles, fingertips, fingers, finger joints, tattoos, veins, elbow, wrist, and higher and lower points on the skin (e.g., [2, 3, 13]). For example, Oh and Findlater [13] found that strategies participants used in creating on-body gestures included using specific parts of the hands - such as fingertips and palm - as landmarks. A system by Gannon et al. [3] allows incorporating individual landmarks, such as knuckles, freckles, veins, and tattoos, in calibrating gestures (which travel through those). Participants found these useful when both hands were engaged in skin input. Dezfuli et al. [2] reported that participants found nine landmarks on the palm side of the hand (five convex and concave areas on the palm and four digit fingers) which they believed they could easily touch without looking. However, these studies focus on landmarks in local areas of the skin (the palm in $[2,13]$ and the forearm in [3]). We could not find previous research that addressed a comprehensive set of the mentioned landmarks over the hand and forearm.

\section{STUDY}

We conducted an in-depth study where participants placed 30 virtual items on their hand and forearm and tried to recall their locations. The overall purpose of the study was to examine:

1. Where participants place items on the hand and forearm?

2. What kind of landmarks they use?

3. What their recall performance will be?

4. What type of strategies participants use when placing items on their skin?

\section{Items}

The 30 items that were used in this study as virtual items were everyday things, collected by searching Wikipedia, and various vocabulary sites, with four categorical words (emotions, buildings and places, occupations, and family members ${ }^{1}$. We used these everyday items for two reasons. First, we aimed to enable creation of personal associations between items and the skin, because when placing virtual items in physical surroundings, creation and use of associations is suggested to improve recall of item locations [15]. Furthermore, people often have personal associations with their computer's

\footnotetext{
${ }^{1}$ The items were: Disgust, Embarrassed, Hopeful, Irritated, Melancholy, Panic, Surprise, Teacher, Actor, Dentist, Farmer, Lawyer, Nurse, Police, Aunt, Brother, Cousin, Daughter, Father, Mother, Nephew, Sister, Bank, Hospital, House, Library, Museum, Park, Restaurant, Supermarket.
} 
contents, such as the mobile phone contact list and applications menu. Second, in order to identify what types of landmarks are used, and to find personal differences in their usage, we aimed to induce creative use of landmarks on the skin. Using items from a particular application, or item groups with an explicit, discrete, or linear order (such as numbers or alphabets), might influence how participants organize the items, limiting the generalizability of the results to other applications.

It was necessary to use an adequate number of items to allow us to examine a variety of placements across the skin. With 30 items, we aimed to induce the participants to use multiple areas and multiple types of landmarks on the hand and forearm, as well as inventive strategies in placing the items. Furthermore, the number needed to be high enough to allow measurement of recall performance and possible learning effects after repetition of recall tasks without ceiling. In Perrault et al. [15], the participants were already in the first phase able to recall nearly all (in average 47.5 out of 48 ) physical locations of virtual items they had placed in a room. We first piloted the setup with 60 items, but recognized that the experiment's duration extended to over 1.5 hours, and that recall rate was low. We aimed to keep the experiment short enough to prevent fatigue, which might reduce participants' motivation to try their best to create placement strategies that would result in effective recall. We therefore piloted the experiment again using 36 items and two participants; recall rates suggested that, with 30 items, participants would be unlikely to achieve full recall of the locations on the skin.

\section{Participants}

Data were collected from 16 right-handed participants (9 females and 7 males, with a mean age of 26.7 years). All of them used a touchscreen mobile phone daily ( 9 of them frequently throughout the day), and 10 participants used a touchscreen tablet device daily. We recruited participants from three different nationalities and five occupations. Participants were instructed to wear a shirt that left the skin bare below the elbow, and to remove all jewelry and watches when participating in the experiment. The experiment lasted approximately an hour, and participants received gifts as compensation for their time.

\section{Study Setup and Implementation}

The purpose of the study was to examine (1) placement of items on the hand and forearm, (2) landmarks on the skin at item locations, (3) recall performance of these locations, and (4) participant strategies for item placement. Therefore, we required three types of data: touch locations on the skin, pictures of the skin features, and interview recordings. Pictures were taken with a camera, and interviews recorded on a mobile phone. Table 1 summarizes the phases of this study, the tasks or actions conducted in each phase, and the purpose or the data collected in those.

To capture the touch locations on the skin, we designed a setup that provided us both real-time finger coordinates, and a representation of the skin surface that enables relating the coordinates to it post hoc. The coordinates needed to be processed in real-time to provide participants with feedback about their recall performance during the tasks - that is, whether they had touched the location at which they had placed an item earlier on. The representation of the skin's surface allowed us to use the finger coordinates to infer actual touch points.

\section{Tracking Touch Locations}

We used an OptiTrack motion-capture system consisting of eight Flex13 cameras with 56 degrees field of view to track the index finger (Figure 2) and deliver the fingertip coordinates to an experimental software. This motion capture system tracked the IR reflective markers on the finger with a $120 \mathrm{~Hz}$ sampling rate, and with less than $0.3 \mathrm{~mm}$ spatial accuracy in 3D space.

We designed and 3D-printed a rigid body (a rigid piece of plastic on which reflective markers are attached) for the index finger (Figure 2). This rigid body was designed to allow

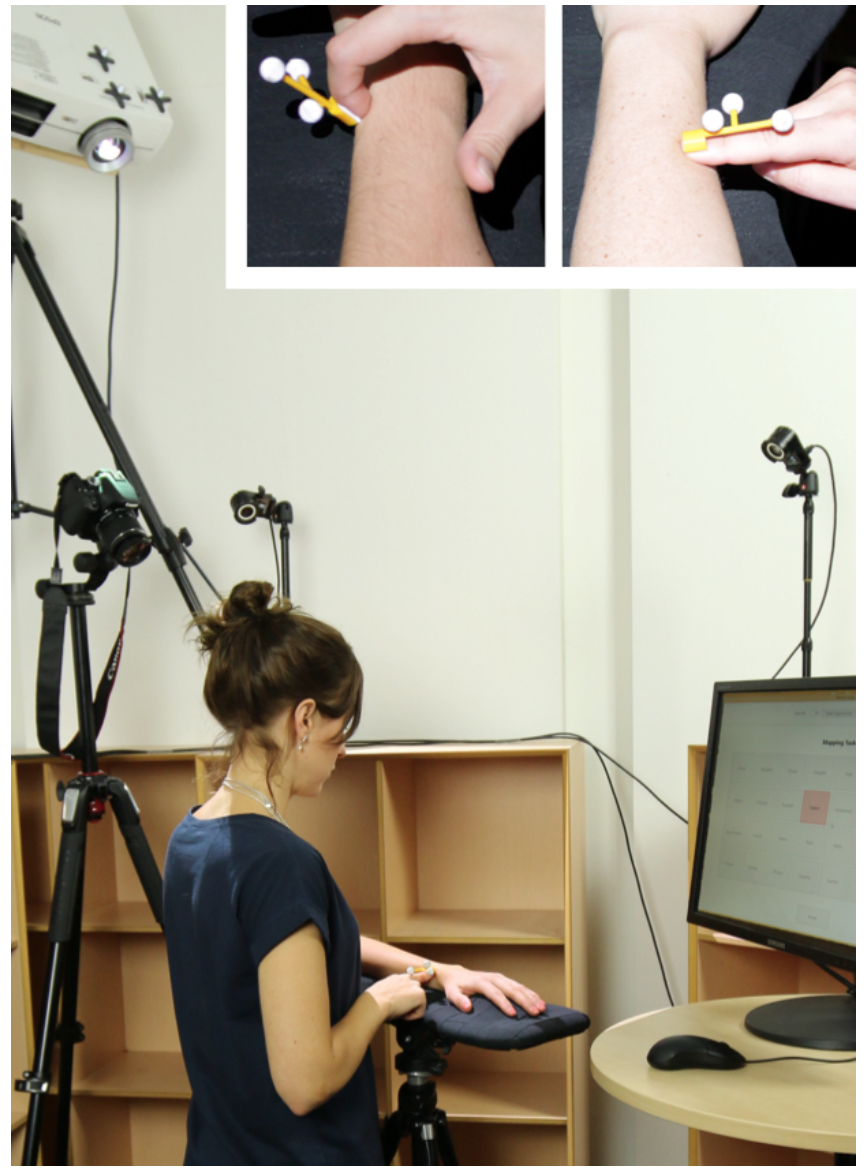

Figure 2: Study setup. The pictures at the top show the yellow rigid body with markers attached to the index finger nail. Participant's left hand is kept in a relaxed posture on the armrest, while the index finger of the right hand is pointing at a location for an item selected on the screen. The projector on the top left corner is used to project a posture calibration grid on the arm. The OptiTrack motion capture system with eight cameras (two of those visible in the picture) is placed around the participant, tracking the rigid body attached to the index finger and delivering its coordinates. A Canon EOS camera placed above and to the left of the user captures pictures of the hands. 
Table 1: Experimental phases, tasks, and data collection.

\begin{tabular}{|c|c|c|c|}
\hline Steps & Phase & Task & Purpose and Data Collection \\
\hline 1 & Posture Calibration & $\begin{array}{l}\text { The experimenter sets the armrest to support a } \\
\text { relaxed posture, then draws the projected grid } \\
\text { with a UV marker. }\end{array}$ & $\begin{array}{l}\text { To mark the posture so that the same posture can be adapted } \\
\text { for all phases. Needed for evaluating whether recall was suc- } \\
\text { cessful. }\end{array}$ \\
\hline 2 & Mapping Phase & $\begin{array}{l}\text { The participant places } 30 \text { virtual items on the } \\
\text { skin. }\end{array}$ & $\begin{array}{l}\text { Location coordinates of items from motion capture data of } \\
\text { the finger, and pictures of the hands placing the items from } \\
\text { a camera. For examining placement of items and landmarks } \\
\text { on the skin. }\end{array}$ \\
\hline $\begin{array}{l}\text { Before } \\
\text { each } \\
\text { phase } \\
\text { 3-8 }\end{array}$ & Break & $\begin{array}{l}\text { The participant adapts the posture to match the } \\
\text { UV marker lines and the projected grid before } \\
\text { beginning the next phase. }\end{array}$ & $\begin{array}{l}\text { Short breaks help the participant to keep still during the } \\
\text { phases. }\end{array}$ \\
\hline 3,5 & Learning Phases & $\begin{array}{l}\text { The participant tries to recall the locations of } 30 \\
\text { virtual items on the skin. }\end{array}$ & $\begin{array}{l}\text { Giving participants feedback of recall performance to induce } \\
\text { learning. Recall performance measures from finger coordi- } \\
\text { nates. }\end{array}$ \\
\hline 4,6 & Recall Phases & $\begin{array}{l}\text { The participant tries to recall the locations of } 30 \\
\text { virtual items on the skin. }\end{array}$ & $\begin{array}{l}\text { No feedback given of recall performance. Recall perfor- } \\
\text { mance measured from finger coordinates. }\end{array}$ \\
\hline 7 & Random Recall & $\begin{array}{l}\text { The participant tries to recall the locations of } 6 \\
\text { randomly chosen virtual items on the skin. }\end{array}$ & $\begin{array}{l}\text { Recall performance measures from finger coordinates. Re- } \\
\text { call in a random order without showing the order used in pre- } \\
\text { vious phases. Allows us to determine whether this affected } \\
\text { recall performance. }\end{array}$ \\
\hline 8 & $\begin{array}{l}\text { Capturing the skin } \\
\text { surface }\end{array}$ & $\begin{array}{l}\text { The experimenter captures } 15000-20000 \text { co- } \\
\text { ordinates across the hand and forearm which } \\
\text { represent the skin surface in a posture that was } \\
\text { used in the experiment. }\end{array}$ & $\begin{array}{l}\text { To relate the location coordinates of the index finger to the } \\
\text { actual skin surface in a posture it was kept during the phases. }\end{array}$ \\
\hline$(2), 9$ & Interviews & $\begin{array}{l}\text { Think-aloud motivations for placements of items } \\
\text { in phase } 2 \text {. Semi-structured interview after the } \\
\text { experiment. }\end{array}$ & $\begin{array}{l}\text { Voice records to gain insights about placement strategies, } \\
\text { and personal associations between the items and the skin. }\end{array}$ \\
\hline
\end{tabular}

robust tracking of participants' fingertip in 3D space, allow natural posture of the finger in pointing, and yet leave the fingertip uncovered. We avoided placing one of the markers on the nail, because due to the size of the sphere (diameter $14 \mathrm{~mm}$ ), it would obscure participants' vision of small landmarks (such as freckles) when touching the skin nearby.

A minimum of three of such spherical markers on the rigid body were needed to robustly track the orientation and coordinates of the finger in $3 \mathrm{D}$ space. This orientation was necessary to determine the fingertip's precise location, because finger posture (e.g., orientation in relation to the skin surface) varies between touch locations across the hand and arm. For example, the index finger is extended when touching the near side of the arm (Figure 2, top right) because flexion would cause the nail to touch, whereas the finger is flexed when touching the far side of the arm (Figure 2, top left). Without orientation, varying approaching angles of touches (e.g., tilting the finger) at a single point would have been mistakenly interpreted as different touch coordinates.

The head of the rigid body was curved similarly to a nail so that it attached tightly to the nail with double-sided adhesive. Attaching the rigid body to the nail left all of the index finger skin on the palm side uncovered, allowing participants to also feel the skin of the left hand when placing items.

\section{Images of the Skin}

A Canon EOS 600D camera was used to capture highresolution pictures of the hands. The pictures were used in analysing landmarks on the skin, and also displayed to participants during the experiment after false touch locations. The camera was placed above the participant to the left (Figure 2) so as to cover the entire left forearm and hand, providing a first person view when these images were displayed as feed- back during the experiment. The purpose of a first-person view was to help participants relate displayed touch locations to their skin. The camera was controlled by an experimental software.

\section{Software}

The software developed for this study streams OptiTrack data to log touch coordinates, processes touch data, controls the camera to capture images, and serves as a user interface in the experimental task.

The interface displayed 30 buttons, each labeled with an item name in a randomised order. The button list was randomised to encourage participants to place the items in an order of their choice instead of a pre-defined order. The list stayed the same throughout the experiment, allowing participants to find items they wanted to select without remarkable effort.

Participants selected items to place or recall by clicking them with a mouse. When touching the skin on the location they selected for an item, the experimenter pressed the space bar and the software captured a picture with the camera and logged the coordinates of the index finger tip from the OptiTrack.

When placing the items, the software ensured that the location did not overlap with any of the previously placed items; if it did overlap, the interface turned the button red so that the participant could select a new location for it. To evaluate recall performance, the software calculated the nearest item from the location of touch to determine if the selection corresponded to the location where the item that was selected on the screen was placed earlier. If the location corresponded to that item, the recall was logged as successful. 


\section{Interpreting Touch Locations on the Skin}

Physical IR-trackable markers (such as those provided by OptiTrack and used on the rigid body on the index finger) would have provided the participants with additional landmarks on the left hand and arm, imposing a risk of bias in the usage of skin-based landmarks. Therefore, the OptiTrack was used only for tracking the coordinates of the index finger. Because a representation of the skin surface was needed, the posture of the left hand and arm had to be consistent throughout the experiment. The participants were instructed to keep their hand still during all phases; the location and shape of the hand were then captured in a separate calibration phase after the experimental phases.

We also built an armrest to assist participants in keeping their hand and arm relaxed and still during the experiment (Figure 2). The armrest was shaped from foam, and contained two prominences on both sides of the arm, one under the wrist, and one hemispherical under the palm. With the design of the armrest we aimed to not provide any extra landmarks around the hand and arm, and therefore also covered the armrest with black fabric. The armrest was placed on a tripod to enable adjusting it according to the participant's height.

To calculate the nearest items based on index finger coordinates only, it was necessary for participants to adapt precisely the same posture each time. Before initiating the experiment, a grid (Figure 3) was projected on the left hand and forearm and its lines were drawn on the skin with a UV marker invisible in normal light. Before each experimental phase, the projector and a blacklight torch making the UV marker visible were turned on, and the posture was adapted until the lines matched and maintained throughout the next phase.

To relate the OptiTrack data of the index finger to the skin, we build a 3D mesh representing the skin surface of each participant. After the tasks, the experimenter used another rigid body to capture points (coordinates) on the skin. The experimenter then "drew" the outlines of the hand and arm and "colored" across the entire skin surface with a rigid body marker. By tracking the marker that traveled on the skin surface, we collected approximately 15-20 000 coordinates on each participant's skin. We exported this point cloud from OptiTrack to MatLab, and performed a Delaunay triangulation to create a mesh representing the skin surface. The index finger coordinates were then plotted on this mesh (see examples of these
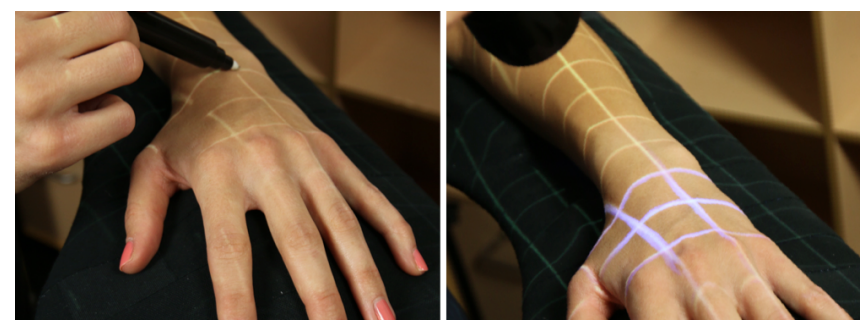

Figure 3: Picture on the left shows the experimenter drawing the projected grid with UV marker invisible in normal light. Picture on the right shows the participant adapting her posture to match the blue UV marker lines and the projected grid before starting the next recall phase. meshes in the Results section), allowing us to analyse touch locations on the skin.

\section{Task}

The task was to place 30 virtual items on the surface of the hand and forearm (skin and nails), and then recall their locations. Participants were free to choose the order in which they placed and recalled these items.

The participants placed and recalled the items one by one by first clicking an item button of their choice with a mouse, and then touching the selected location on the skin using their index finger (Figure 2). The selection's coordinates were captured when the participant told the experimenter to confirm the location (e.g., saying "there"). This verbal command was used to avoid any additional body movements of participants and possible errors in tracking precise touch locations caused by those. Moreover, this allowed participants to view and feel their skin before making a selection; this would not be possible with, for instance, a capacitive touch sensor on the fingertip.

\section{Procedure}

The experiment consisted of six phases, including mapping, learning, recall, and a random recall phase (see Table 1). The mapping phase was performed first, in which the participants placed the items onto the skin. In the learning phase participants trained this mapping with a recall task by receiving feedback on their selections. In the recall phase participants attempted to recall all locations without any feedback on their performance. These learning and recall phases were performed twice to reveal possible learning effects. Finally, we performed a short random recall test presenting six items, one after the other. The purpose of this recall test was to examine whether recall order had any effect on recall performance by preventing participants from following a self-chosen sequential and spatial order and forcing them to follow a random one instead.

\section{Posture Calibration}

Participants were instructed to keep their hand in the same fixed but relaxed posture throughout the phases. This posture was calibrated at the beginning of the experiment by adjusting the armrest to a height where the arm and hand were fully supported, and where participant felt relaxed. The projected lines were then drawn on the skin, and before each phase this posture was re-adapted.

\section{Mapping Phase}

In the mapping phase participants were instructed to place the items anywhere on the visible skin or nails between the elbow and the fingertips. After successful placement of an item, that item's button was disabled so that the participant saw which items remained for placement. Once an item was selected and placed, no further changes to the mapping were allowed. Therefore, participants were instructed to take their time while proceeding through the item list, and to try to be inventive with their choice of locations so that they could more easily recall them later on. We also asked the participants to 'think aloud' while mapping to gain insights on their 
strategies. Their voices were recorded during the mapping phase so as to capture these thoughts.

To separate successful recalls from false ones by calculating the nearest items, we needed to prevent overlapping item placements. If the item distance to the nearest item placed earlier during mapping phase was smaller than the $8 \mathrm{~mm}$ threshold, the item button turned red, and the participant could place the item elsewhere. The threshold was twice the mean error $(4 \mathrm{~mm})$ of touches in a pilot in which a participant pointed to 10 visual targets marked on the skin. The $8 \mathrm{~mm}$ threshold also coincides with approximately half of the width of an average adult index finger tip [4].

\section{Learning Phases}

In the learning phase participants were shown the same items in the same order as in the mapping phase. The learning phase consisted of a recall task with feedback. Using the recall task allowed performance to be measured, while simultaneously inducing learning. The items and their location were selected in a similar procedure to that used in the mapping phase, by clicking the item button with a mouse and touching its recalled location on the skin.

Recall was considered to be correct if the nearest point (Euclidian distance in 3D space) from the 30 locations determined in the mapping phase was the location of the selected item and the distance to that was within $20 \mathrm{~mm}$. When the recalled location was correct, the item button turned grey (i.e., was disabled). ). If the location was incorrect, an image of the participant's hand and the finger touching the selected location (captured in the mapping phase) was displayed on the screen (see an example of such image in Figure 5). With this visual guidance, the participant then reattempted to select the correct location.

\section{Recall Phases}

In the recall phases the task was similar to the learning phase. However, participants received no feedback as to whether the recalled location was correct or not. After selection, the button was disabled.

\section{Random Recall}

The random recall phase consisted of six randomly selected items from the item list used in the experiment. The interface displayed the items one at a time centered on the computer's display. This prevented participants from using the item's position on the display, or the sequential order of items, to aid recall. Otherwise, the task was similar to the one used in the recall phase.

\section{Interviews}

After the recall tasks, we interviewed participants using a semi-structured approach. For the interview, participants were shown the same list of items they had seen during the experiment. They were then asked to describe their strategies for item placement on the skin. The experimenter also asked first, whether the participant grouped the items and if so, how; second, how they chose the locations on the skin, and whether they had used any landmarks there; and finally, were some item locations easier or more difficult to recall and if so, why.

\section{RESULTS}

Touch data and pictures were used for examining where the participants placed items on the hand and forearm. Based on the meshes and the associated touch coordinates, we coded touch locations on physical areas and anatomical landmarks, such as bones, joints, and nails. The pictures complemented the touch data - which does not extend to purely visual features, such as tattoos or pigments - and also helped to resolve uncertainties regarding touch locations. Touch data was used to calculate participants' overall recall performances, as well as recall performances per items. The coding of landmarks was used for calculating recall performances on specific areas and landmarks. Finally, voice recordings from mapping phases and interviews were used for exploration of personal associations between items and locations on the skin, as well as strategies used to organize and place the items.

The total duration of the experimental phases from the beginning of the mapping phase to the end of random recall was 42 minutes, on average. The mapping phase took an average of $10.8 \mathrm{~min}$, the first learning phase $7.1 \mathrm{~min}$, the first recall 4.1 $\mathrm{min}$, the second learning phase $5.3 \mathrm{~min}$, the second recall 3.0 min, and the random recall 31.9 seconds.

\section{Placement of Items}

We coded the placement of items from the touch coordinates plotted on the meshes of hand surfaces together with the pictures taken during the mapping phase. Examples of these two data for P2 are depicted in Figure 5. Coding was conducted by examining the plots and images, and creating new landmark types when they emerged.

We began the coding by dividing the skin to five areas: Fingers, Hand, Wrist, Arm, and Elbow. Out of all items, 39.4\% were placed on fingers, $16.5 \%$ on the hand, $6.0 \%$ on the wrist, $35.2 \%$ on the arm, and $2.9 \%$ on the elbow. We then coded

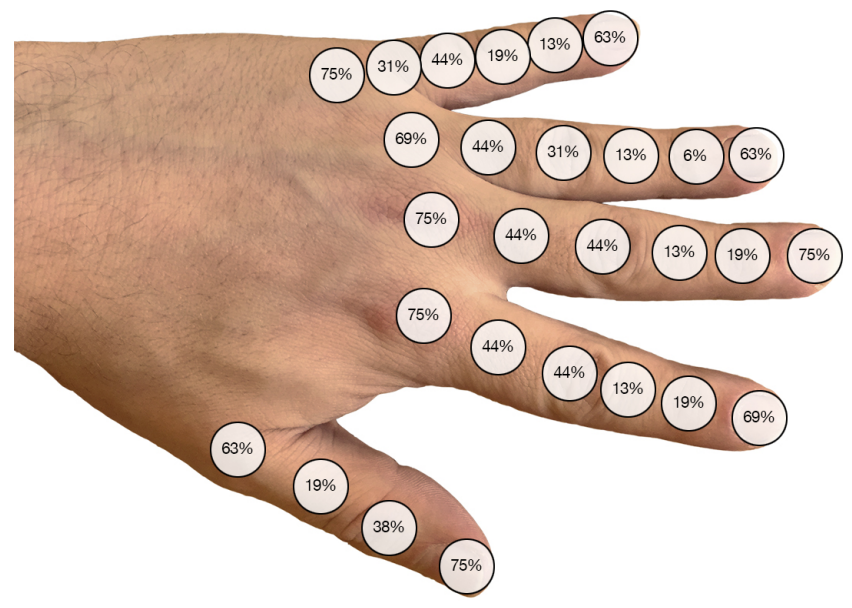

Figure 4: Location frequencies by a percentage of participants who placed an item there. We coded six locations from the nail to knuckle: 1. Distal phalanx (nail), 2. Distal interphalangeal (DIP) joint, 3. Intermediate (middle) phalanx, 4. Proximal interphalangeal (PIP) joint, 5. Proximal phalanx, and 6. Metacarpophalangeal (MCP) joint (knuckle). The thumb lacks an intermediate phalanx, and therefore has no DIP joint. 

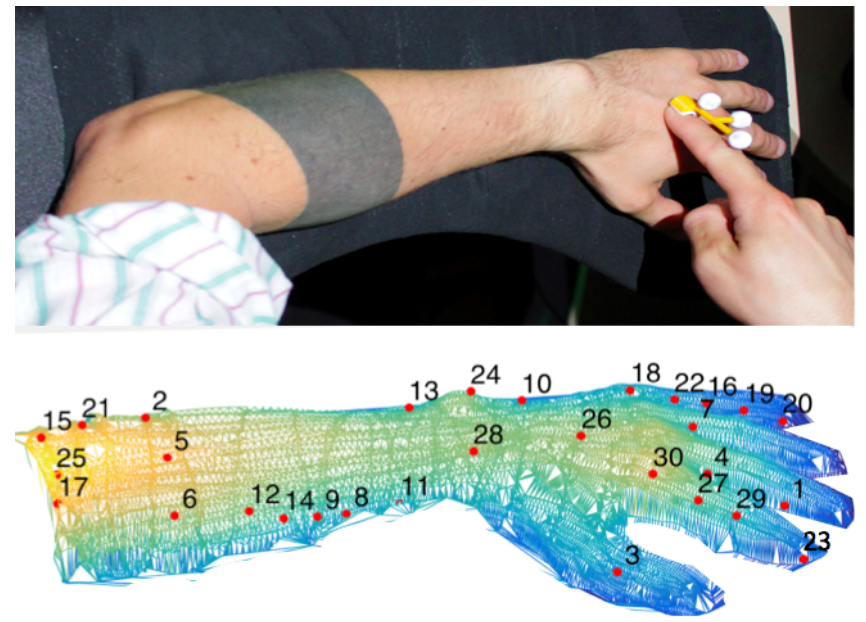

Figure 5: An image of the skin and the mapped item locations plotted on the mesh of P2. P2 is selecting a location on a vein on the back of the hand. The three item IDs $(2,5$, and 6$)$ placed over the midline of the tattoo are plotted on the mesh, and correspond to 'Embarrassed', 'Melancholy', and 'Panic', respectively.

the item locations on the fingers for each finger separately by dividing it into joints and phalanges. (Phalanges are the fingerbones between the joints). Most participants placed items on nails (fingertips) and knuckles (ranging between 63\% and $75 \%$ in each case), whereas on other joints and phalanges the frequencies were more distributed (Figure 4). The participants never placed more than 6 items on a finger, each on a separate bone or joint. Therefore, no other landmarks were recognized on the fingers.

We found seven additional landmark types outside the fingers. One type is close to the fingers; a soft area on the hand between the carpometacarpal bones of the thumb and index finger. Out of all items, $1.9 \%$ were placed there. About 1.25\% of all items were placed on the pisiform (the bone on the wrist visible on P2 in Figure 5). Another anatomical landmark was the wrinkle or line at the anterior side of the elbow, which is visible when the elbow is flexed. Out of all items, 3.5\% were placed here. Birthmarks, moles, or freckles were used for $7.1 \%$ of the items. Tattoos were used for $2.3 \%$, veins for $1.0 \%$, and scars for $0.2 \%$ of the items.

\section{Items on Specific Locations}

We also examined the frequency of items on landmarks and areas on the skin. Family members were most often (69.5\%) placed on fingers. Most participants (81.3\%) placed 'Father' on fingers and $75.0 \%$ also placed 'Mother' and 'Daughter' there. Emotions and places were often placed on the forearm. For example, most participants placed 'Melancholy' (81.3\%) and 'Embarrassed' (62.5\%) there. Separating specific landmarks within these areas, we found that almost half of the participants (43.8\%) placed 'Mother' and 'Father' on the fingernails, and $31.3 \%$ also 'Brother' and 'Sister'. Three participants had tattoos and all of them placed 'Melancholy' on those. Most of the items placed on tattoos (88.9\%) were emotions.

\section{Recall Performance}

The average performance in the first recall phase was 19.8 $(.95 \mathrm{CI} \pm 2.8)$ items and in the second recall phase $21.2(.95$ $\mathrm{CI} \pm 3.0)$ items. Although a slight trend of increased performance after learning phases can be seen (Figure 6), there was no significant improvement after repetition.

\section{Learning}

Visual guidance (images shown after erroneous selection in learning phases) helped in learning; $60.6 \%$ of the cases in the first learning phase and $52.8 \%$ of the cases in the second learning phase resulted in the correct selection when retrying. However, when participants did not recall the location even after receiving visual help (when retrying), in most cases they were not able to recall the locations of these items in later phases either $(72.3 \%$ of the fails after visual help in the first learning phase were failed in the first recall phase, and $64.7 \%$ of the fails in the second learning phase also failed in the second recall phase). Similarly, when participants succeeded after help, they also recalled well in later phases, that is, learnt the items they recalled. This suggests that using landmarks is important for performing well in recall; even when people are shown where their finger pointed, they still cannot find the exact location without having some form of reference point on the skin.

\section{Random Order}

There were no significant difference between participants' average recall performance in the random recall phase (where $67.7 \%, .95 \mathrm{CI} \pm 14.3 \%$ of item locations were recalled correctly) and in four learning and recall phases (where $68.1 \%$, $.95 \mathrm{CI} \pm 9.2 \%$ of item locations were recalled correctly). Thus, there is no evidence that sequential ordering of item selections in the experiment (which the participants were free to choose) and the spatial layout of items on the display (which was randomized) have affected recall performance. This suggests that usage of landmarks, item locations on the skin, and personal strategies in mapping the items might influence recall more than selection order or layout of the reference interface.

\section{Items}

Each item category contained items that were easier to recall than others. Among the emotion items, the location of 'Hopeful' was recalled significantly more often $(79.7 \%, .95 \mathrm{CI}$ $\pm 11.1 \%)$ than the locations of 'Embarrassed' $(65.6 \%, .95 \mathrm{CI}$ $\pm 16.0 \%)$, 'Irritated' $(60.9 \%, .95 \mathrm{CI} \pm 15.4 \%)$, and 'Melancholy'(62.5\%, $.95 \mathrm{CI} \pm 16.9 \%)$. Among occupations, 'Nurse' resulted in significantly better recall performances $(78.1 \%$, $.95 \mathrm{CI} \pm 11.8 \%)$ than 'Lawyer' (59.4\%, $.95 \mathrm{CI} \pm 18.1 \%)$. In the family category, the location of 'Mother' was significantly more often recalled $(78.1 \%, .95 \mathrm{CI} \pm 16.8 \%)$ ) than 'Daughter' (56.3\%, .95 CI $\pm 19.2 \%)$. Among places, 'Hospital' (76.6\%, $.95 \mathrm{CI} \pm 12.4 \%)$ and 'House' $(76.6 \%, .95 \mathrm{CI} \pm 14.2 \%)$ were recalled significantly more often than 'Bank' $(60.9 \%, .95 \mathrm{CI}$ $\pm 17.5 \%)$ and 'Library' $(57.8 \%, .95 \mathrm{CI} \pm 18.0 \%)$. Although these differences were found between single items, there were no difference in recall between item categories. This suggests that regardless of the types of items, personally important ones, which the users can associate with, are recalled better. 


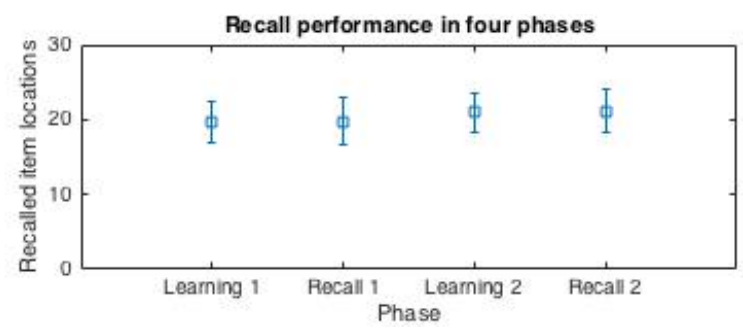

Figure 6: Recall performance in four phases.

\section{Locations}

Item locations in the second recall phase were recalled worse on the arm $(63.3 \%, .95 \mathrm{CI} \pm 12.6 \%)$ than on the fingers $(73.5 \%, .95 \mathrm{CI} \pm 14.9 \%)$, hand $(75.6 \%, .95 \mathrm{CI} \pm 23.9 \%)$ wrist $(75.9 \%, .95 \mathrm{CI} \pm 38.5 \%)$, and elbow $(85.7 \%, .95 \mathrm{CI} \pm 52.2 \%)$. However, as the variations were large, these differences are not significant.

The recall rate of item locations on the nails was $78.0 \%(.95$ $\mathrm{CI} \pm 34.1 \%)$ and on the knuckles $64.7 \%$ (.95 CI $\pm 31.4 \%)$. Items placed on scars and on the pisiform (the bone on the wrist) were recalled in the second recall phase at a rate of $100 \%$, on tattoos at a rate of $82 \%$, on the spot between index and thumb at a rate of $77.8 \%$, and on pigments at a rate of $73.5 \%$. Use of these landmarks, however, was so rare among our participants that statistical significances of recall performances cannot be evaluated.

\section{Qualitative Insights on Placing and Recalling Items}

Table 2 shows a summary of 177 interview snippets. From these, we extracted comments about strategies for placing and recalling items to create groups. As the intention with this data was to complement the image and touch location data, we ignored comments and strategies that would be bettered covered by those sources of data (e.g., where the items were placed, or how groups were placed together).

\section{Selecting Overall Mapping}

The snippets revealed four strategies participants used to lay out their overall mappings between items and their arm and hand. A mapping works as a principle for relating one or more items to the hand and arm. Most participants combined multiple mappings, either because different categories required different mappings or because one mapping stopped working (e.g., running out of space on a finger).

First, 10 participants speak about absolute spatial features and how these work to structure item placement. They mentioned creating lines and super imposing them on their arms (4 participants); as one person explained, "I had lines in many places, like imaginary lines. So I had partly tattoos, injury, knuckles, and then partly imaginary lines." (P16). Four other participants discussed distances "I tried to put things next to each other and to kind of equal distances." (P10). Some participants also spoke about creating a "mental map", a "grid", or a "spatial ordering". In all cases, space rather than landmarks was the guiding principle.
Second, 6 participants decided to map particular groups of items to particular areas. Those areas could be anatomical (e.g., "I'm trying to keep the emotions on the fingers," P1), easy to access ("The front of my arm, the first part, is kind a handy, easy access, so I picked the places I like the most," P7), or importance (e.g., "And the thumb I choose for important stuff, like hospital or being sick, like you have to call a dentist or nurse.” P8). All of these give a clear mapping of items to particular areas.

Third, two participants used the sense of touch on its own. One of them explained: "So, I kind of went on feeling that I could remember the touch, like where I touched my skin. And that kind of helped too, so it was a mix of like an emotional or image association, and if it felt right, if it felt like the right place to touch." (P13).

Fourth, all participants on at least one occasion proceeded with an anchor and association mapping, meaning that they chose one item to serve as an anchor and then proceeded to place items around that anchor. P9 started from a birthmark: "Museum, let's check this birthmark. So museum.. Library. Supermarket here. Park right next to it. So I have this tiny square right next to my birthmark."

\section{Relating Items to Each Other}

In both placing and recalling items, participants took care to relate items to other items. As mentioned above (in anchoring), some participants derived the bulk of their mapping in this way. Next we summarize the different ways of doing so; typically these do not relate to landmarks or skin features.

First, participants mentioned many principles of ordering. These included alphabetical ordering, and ordering based on size or age. One participant also used a process order (P16): "Then the food stuff, I kind a drew line across my arm. And I grouped those in a process order, so first there is a farm and then a supermarket."

Second, three participants talked about creating stories when placing or recalling items. For instance, one participant placed items while making up a story (P14): "Okay, so once upon a time there was a house (to index finger nail), and the house was located in a park (proximal joint). And there lived a farmer (index finger's knuckle). Who was very embarrassed (middle finger's knuckle), because he bought all his groceries in a supermarket (proximal joint) and sold those to a restaurant (middle finger nail)."

Third, a number of different types of personal associations also served as ways of relating items within an area. For example, P2 used a perceived order of value: "Like I placed the bank on the tip because that's most prestigious and rich and going down to the supermarket."

\section{Coupling Items to Skin Features}

One key tactic in item placement is the association of a particular item with a feature of the skin. We differentiate skin features that are personal (e.g., a scar) from those that are shared (e.g., knuckles). An orthogonal dimension, then, depends on whether the reason for coupling an item to a feature is objective or based on a personal experience. This provides 
Table 2: Grouping of the interview data, the number of participants who mentioned these strategies, description of the strategies, and example quotes from snippets.

\begin{tabular}{|c|c|c|c|}
\hline & $\mathbf{N}$ & Explanation & Examples \\
\hline \multicolumn{4}{|l|}{ Finding layout } \\
\hline Spatial mapping & 10 & $\begin{array}{l}\text { Using mental maps, imaginary lines, } \\
\text { and distances along those. }\end{array}$ & $\begin{array}{l}\text { "I used distances also to remember where I put items. Like spatial order- } \\
\text { ing." (P7) }\end{array}$ \\
\hline Mapping onto an area & 6 & $\begin{array}{l}\text { Deciding to place a group of items } \\
\text { on an area. }\end{array}$ & $\begin{array}{l}\text { "Well basically I started with family members because family is important } \\
\text { so I used them as in the primary part of the hand like fingernails." (P4) }\end{array}$ \\
\hline Feeling a way forward & 2 & $\begin{array}{l}\text { Touching the skin and finding loca- } \\
\text { tions by touch. }\end{array}$ & $\begin{array}{l}\text { "I also used finger structure, so I was trying to feel bones and spaces } \\
\text { between those." (P11) }\end{array}$ \\
\hline Anchoring to a point & 16 & $\begin{array}{l}\text { Finding a starting location and mov- } \\
\text { ing forward in an ad hoc fashion. }\end{array}$ & $\begin{array}{l}\text { "Then I had like mental buttons. I made a grid. I started from the birth- } \\
\text { mark as a corner and then from there. It made easy to remember." (P14) }\end{array}$ \\
\hline \multicolumn{4}{|l|}{ Organizing items } \\
\hline Common & 4 & $\begin{array}{l}\text { A commonly used ordering, e.g., } \\
\text { alphabetical ordering, or ordering } \\
\text { based on size. }\end{array}$ & $\begin{array}{l}\text { "Alphabetical order I used for emotions, because only hopeful was re- } \\
\text { motely good so I couldn't group those like good and bad feelings, so then } \\
\text { I just didn't see any way to differ between various bad feelings. So the } \\
\text { alphabetical order worked just fine for me." (P7) }\end{array}$ \\
\hline Stories & 9 & Fictional and personal stories. & $\begin{array}{l}\text { "So mum was a nurse so nurse next to it. And my dad is in the hospital, } \\
\text { so I put hospital above him. Then next to them my sister, and my sister is } \\
\text { a lawyer, so that above her. And then my brother. And he wanted to be a } \\
\text { policeman so that's above him." (P14) }\end{array}$ \\
\hline Associations & 6 & $\begin{array}{l}\text { Ordering based on personal percep- } \\
\text { tion of a hierarchy. }\end{array}$ & $\begin{array}{l}\text { "Then I had the family group, which I put close family on one finger in } \\
\text { some kind of hierarchy with mother on top and the sister in the bottom } \\
\text { because thats how my family is." (P2) }\end{array}$ \\
\hline \multicolumn{4}{|l|}{ Coupling Items to Skin } \\
\hline $\begin{array}{l}\text { Shared feature and } \\
\text { personal experience }\end{array}$ & 10 & $\begin{array}{l}\text { Connecting a personal experience } \\
\text { to a shared landmark, such as to a } \\
\text { knuckle. }\end{array}$ & $\begin{array}{l}\text { "The reason why I placed the dentist towards my elbow, is because when } \\
\text { I went to the dentist once, I hit my elbow and we made fun with the dentist } \\
\text { about that." (P9) }\end{array}$ \\
\hline $\begin{array}{l}\text { Personal feature and } \\
\text { personal experience }\end{array}$ & 8 & $\begin{array}{l}\text { Connecting a personal story to a } \\
\text { personal feature, such as to a tattoo } \\
\text { or birthmark. }\end{array}$ & "Tattoo is like a mourning band for me so I put melancholy there." (P2) \\
\hline $\begin{array}{l}\text { Shared feature and } \\
\text { common experience }\end{array}$ & 5 & $\begin{array}{l}\text { Common connections, such as cul- } \\
\text { tural associations. }\end{array}$ & $\begin{array}{l}\text { "Disgust: Disgust I'll place here. Because if you are disgusted you might } \\
\text { give someone the finger." (P3) }\end{array}$ \\
\hline $\begin{array}{l}\text { Personal feature and } \\
\text { common experience }\end{array}$ & 4 & $\begin{array}{l}\text { Connections other people could } \\
\text { make, but to a personal landmark. }\end{array}$ & $\begin{array}{l}\text { "Irritated: Irritated because I have an injury on my arm, and it's very irri- } \\
\text { tated right there." (P3) }\end{array}$ \\
\hline
\end{tabular}

four main groups of reasons for coupling items to skin features.

The most common of these was the association of a shared feature with a personal experience (10 participants). Reasons for placing the item mother include "Put it here because the wrist is my favorite part of my arm and it's the one that I touch the most. And if this would be to call my mother, this would be the easiest one," (P1); "There, I'm placing mother on the index finger, because she's to me, mother to me is very, she's given me directions. Sort of like pointing me the right direction," (P13); and "I place mother here, because it's like a very soft spot on my arm and I like to connect that with mother." (P3). These, and many other associations, centered on using a personal story (such as P2's: "The ring finger is for the wedding ring and sometimes the proposal is a surprise") to connect common features (e.g., ring finger) with items (e.g., surprise).

The personal story is often linked to a personal feature (8 participants), such as a vein, tattoo, or birthmark. Emotions were linked to veins in three comments, for instance, 'I'm gonna start with panic, and I'm gonna click right under my index finger, and it's because I have a little vein there," (P6); "Panic: I place panic here, because I have a big vein here and if I panic it might be like irritated," (P3); and the same participant continuing with "Embarrassed: Because when you are embarrassed you get like hot and I get very sweaty. And I have a vein there who always bumps when I get embarrassed."
Common stories can be linked to common features, such as areas on the hand. Two participants mentioned a connection between thumbs and positive feelings, and two linked negative feelings to the middle finger. For example, "Irritated I put on the bad finger, which you can signal to people that you are irritated with them," and "Hopeful: Hopeful I will put on my thumb. Thumbs up is a kind of positive feeling," (P2).

Finally, common connection can also be linked to a personal feature. For instance, "Hospital... Well that should go to some scar tissue as well, that makes sense" (P12).

\section{Summary of Findings}

We found that participants frequently placed virtual items on both the fingers $(39.4 \%)$ and the forearm (35.2\%). Participants recalled locations of 21.2 items in average (out of 30 , $70.7 \%$ ) when they were allowed to place items themselves, both when traversing a list of all items and when recalling items at random. No significant effect was found between item locations on recall rates, but locations on the arm appear to be harder to recall than on other areas. Item locations on fingernails were well recalled $(78 \%)$, but certain personal landmarks helped to achieve better recall rates, such as scars $(100 \%)$ and tattoos $(82 \%)$. The findings from the effects of visual guidance suggest that landmarks are important for recall even when visual feedback about the item location is given on external display. However, most of the participants used also mental grids alongside other mapping strategies. 


\section{DISCUSSION}

We have presented a study of placing and recalling virtual items on the skin that combines data from motion tracking, images of the skin, and interviews. The results show the complexity and inventiveness of user-generated layouts of items on the skin. While earlier studies have allowed users to place items on their own, the item sets have had natural orderings (e.g., numbers [11], alphabet [17], or directional keys [2]). In the present study, we used everyday items that enabled finding a variety of mapping strategies between and within items and landmarks. We also coupled the use of landmarks to recall performance and showed how particular areas of the hand (e.g., nails) helped participants to achieve higher recall performance.

\section{Implications for Skin Interfaces}

Our study has implications for skin interfaces beyond providing basic knowledge about recall rates. Most importantly, participants used an overwhelming variety of mapping strategies and landmarks; while not all of these would likely be used with an actual interface, recognition of their existence can be beneficial in interface design.

First, our findings on learning effects suggest that using landmarks in mapping is important for recalling item locations on the skin, because without landmarks the exact locations are difficult to retrieve even when visual guidance is provided. These results are in line with previous research: Gustafson et al. [5], for instance, showed that touch accuracy decreases the futher the target is located from a landmark. Our results, however, also suggested frequent use of mental grids. These can be efficient when the items are not located too closely together. The number of items in mental grids per anchor point may also affect recall performance of exact item locations. Therefore, interface designs should allow enough landmarks on the skin to act as anchoring points, and enough distance between virtual items when anchors are absent or mental grids are preferred.

Second, the findings provide insight into the many strategies that are used in coupling items to skin. We found couplings between shared features and common experiences similar to those identified in earlier work. Weigel et al. [18], for instance, have previously reported findings regarding the use of the thumb for positive emotions (because of "thumbs up"), or anterior side of the forearm for personally significant items due to that location's physical proximity to the body. We found that personal experiences can also be connected with common landmarks, and common experiences with personal landmarks. While common landmarks can be used in interface design, the layouts cannot rely on personal ones, such as on tattoos, because not all users will have them. However, many of the personal landmarks are also common among people, such as veins, although these are located on different places on the skin. Therefore, it is important for interface design to recognize which landmarks should be embedded as item locations, and to allow flexible locations for these landmarks.

Third, our results indicate the touch locations participants most frequently used for virtual items. These touch loca- tions can inform technological development of sensors tracking touch input on the skin. For example, our findings suggest frequent use of the fingers and forearm for virtual items, while previous work on gestures [18] have found more frequent usage of the forearm than of the fingers $(50 \%$ vs. $7.3 \%)$. These findings may imply the importance of tracking touch on both the fingers and forearm, while tracking the forearm appears to be more important for gesture input on the skin. Motion capture systems [7] and some systems sensing acoustic signals propagating on the skin $[8,12]$, have already demonstrated their ability to track touches across the hand and arm, and might therefore be suitable for skin interfaces with virtual items.

\section{Limitations and Methodology}

In studies of recall of items (e.g., [15]) the type and number of items placed is a key decision. The number of 30 items were based on pilot studies to avoid ceiling (perfect recall) or floor effects. Future work should study how to increase that number; for instance, by explicitly training participants in the mapping strategies identified in this study.

Recall may also depend, in a non-linear way, on the number of items: as a participant is required to place more items, recall can decrease progressively, for instance because of interference. Thus, we are not suggesting that the average recall of $70.7 \%$ would scale to other numbers of items, but merely observing that in the present setup participants were able to recall around 21 items out of 30 , much more than most studies of skin input have investigated $[2,6,5,7,11,12,13,14]$.

The instructions for recall can have a large effect on performance; we used the approach from [15] of showing an item list and having participants to place and recall while the list was visible. Our empirical approach and the selecetion of items aimed not to restrict the usefulness of results to any particular technology for tracking input, or to any existing interface design that could be mapped to the skin. In the random recall phase, in which participants recalled items without the cue of the list, they performed as well as with the list. For us, this clears up the potential methodological confound that the list may help recall (by associations between its spatial structure and the locations of items, and also by the sequence of recalls).

We recommend that future work addresses item placement around the hand and forearm and allow participants to move their arm (which they will in real recall circumstances); unfortunately, technical limitations prevented this setup for our study. In addition, we only investigated discrete touches; the use of gestures on the skin [18] may enable other recall strategies.

\section{ACKNOWLEDGMENTS}

This project has received funding from the European Research Council (ERC) under the European Union's Horizon 2020 research and innovation program (grant agreement 648785). The work was supported by KAUTE Foundation and Ulla Tuominen Foundation. 


\section{REFERENCES}

1. Chan, L., Chen, Y.-L., Hsieh, C.-H., Liang, R.-H., and Chen, B.-Y. Cyclopsring: Enabling whole-hand and context-aware interactions through a fisheye ring. In Proceedings of the 28th Annual ACM Symposium on User Interface Software \& Technology, ACM (2015), 549-556.

2. Dezfuli, N., Khalilbeigi, M., Huber, J., Özkorkmaz, M., and Mühlhäuser, M. PalmRC: leveraging the palm surface as an imaginary eyes-free television remote control. Behaviour \& Information Technology 33, 8 (2014), 829-843.

3. Gannon, M., Grossman, T., and Fitzmaurice, G. Tactum: A Skin-Centric Approach to Digital Design and Fabrication. In Proceedings of the 33rd Annual ACM Conference on Human Factors in Computing Systems, ACM (2015), 1779-1788.

4. Garrett, J. W. The adult human hand: some anthropometric and biomechanical considerations. Human Factors: The Journal of the Human Factors and Ergonomics Society 13, 2 (1971), 117-131.

5. Gustafson, S., Bierwirth, D., and Baudisch, P. Imaginary interfaces: spatial interaction with empty hands and without visual feedback. In Proceedings of the 23nd annual ACM symposium on User interface software and technology, ACM (2010), 3-12.

6. Gustafson, S. G., Rabe, B., and Baudisch, P. M. Understanding palm-based imaginary interfaces: the role of visual and tactile cues when browsing. In Proceedings of the SIGCHI Conference on Human Factors in Computing Systems, ACM (2013), 889-898.

7. Harrison, C., Benko, H., and Wilson, A. D. OmniTouch: wearable multitouch interaction everywhere. In Proceedings of the 24th annual ACM symposium on User interface software and technology, ACM (2011), 441-450.

8. Harrison, C., Tan, D., and Morris, D. Skinput: appropriating the body as an input surface. In Proceedings of the SIGCHI Conference on Human Factors in Computing Systems, ACM (2010), 453-462.

9. Knibbe, J., Martinez Plasencia, D., Bainbridge, C., Chan, C.-K., Wu, J., Cable, T., Munir, H., and Coyle, D. Extending interaction for smart watches: enabling bimanual around device control. In CHI'14 Extended Abstracts on Human Factors in Computing Systems, ACM (2014), 1891-1896.
10. Lim, S.-C., Shin, J., Kim, S.-C., and Park, J. Expansion of Smartwatch Touch Interface from Touchscreen to Around Device Interface Using Infrared Line Image Sensors. Sensors 15, 7 (2015), 16642-16653.

11. Lin, S.-Y., Su, C.-H., Cheng, K.-Y., Liang, R.-H., Kuo, T.-H., and Chen, B.-Y. Pub-point upon body: exploring eyes-free interaction and methods on an arm. In Proceedings of the 24th annual ACM symposium on User interface software and technology, ACM (2011), 481-488.

12. Mujibiya, A., Cao, X., Tan, D. S., Morris, D., Patel, S. N., and Rekimoto, J. The sound of touch: on-body touch and gesture sensing based on transdermal ultrasound propagation. In Proceedings of the 2013 ACM international conference on Interactive tabletops and surfaces, ACM (2013), 189-198.

13. Oh, U., and Findlater, L. Design of and subjective response to on-body input for people with visual impairments. In Proceedings of the 16th international ACM SIGACCESS conference on Computers \& accessibility, ACM (2014), 115-122.

14. Oh, U., and Findlater, L. A Performance Comparison of On-Hand versus On-Phone Nonvisual Input by Blind and Sighted Users. ACM Transactions on Accessible Computing (TACCESS) 7, 4 (2015), 14.

15. Perrault, S. T., Lecolinet, E., Bourse, Y. P., Zhao, S., and Guiard, Y. Physical Loci: Leveraging Spatial, Object and Semantic Memory for Command Selection. In Proceedings of the 33rd Annual ACM Conference on Human Factors in Computing Systems, ACM (2015), 299-308.

16. Prätorius, M., Valkov, D., Burgbacher, U., and Hinrichs, K. DigiTap: an eyes-free VR/AR symbolic input device. In Proceedings of the 20th ACM Symposium on Virtual Reality Software and Technology, ACM (2014), 9-18.

17. Wang, C.-Y., Chu, W.-C., Chiu, P.-T., Hsiu, M.-C., Chiang, Y.-H., and Chen, M. Y. PalmType: Using palms as keyboards for smart glasses. In Proceedings of the 17th International Conference on Human-Computer Interaction with Mobile Devices and Services, ACM (2015), 153-160.

18. Weigel, M., Mehta, V., and Steimle, J. More than touch: understanding how people use skin as an input surface for mobile computing. In Proceedings of the SIGCHI Conference on Human Factors in Computing Systems, ACM (2014), 179-188. 